\author{
Accelerator Division \\ Alternating Gradient Synchrotron Department \\ BROOKHAVEN NATIONAL LABORATORY \\ Upton, N.Y. 11973 \\ Accelerator Division \\ Technical Note
}

AGS/AD/Tech. Note No. 357

\title{
DECOUPLED BEAM LINE TUNING
}

T. Roser

January 3, 1992

\begin{abstract}
Accurate matching of the beam ellipse in both horizontal and vertical direction is important for successful injection of multiple turns into an accelerator. However, unlike steering corrections the beam ellipse parameters are difficult to measure and therefore tuning is a lengthy process and often based on trial and error. A two-step method is presented here that should allow for a faster and more systematic tuning process. The first step consists of measuring the beam ellipse parameter using either several beam profile monitors or several beam profiles measured with the same monitor but with different settings of the beam transport elements. Once an approximate match has been achieved a tuning procedure based on small changes of the beam line quadrupoles is required. Changes of one individual quadrupole will affect all beam ellipse parameters in both horizontal and vertical direction. The goal is then to combine the affect of changes of several quadrupoles in such a way that for example only the beam parameters in the horizontal plane are affected and the beam parameters in the vertical plane remain unchanged. Generally this is a nonlinear problem and typically requires a least square minimization procedure ${ }^{1}$. If only small changes of the quadrupoles are anticipated the problem can be linearized. The coefficients that relate the changes in the quadrupoles can then be used to define a 'virtual tuning knob' to directly control the correct combination of quadrupoles. In the case of thin lense approximation for the quadrupoles simple analytic formulas for these coefficients can be found. It turns out that in most cases the coefficients obtained by the thin lens approximation already result in very good decoupling.
\end{abstract}

A general transport matrix is fully determined by the choice of two sets of three independent parameters for the horizontal and vertical plane, respectively. Therefore, the requirement that the beam parameters in one plane remain unchanged results in three conditions for the elements of the transport matrix. These conditions can be met with three quadrupoles. In order to be able to make changes of the beam parameter at least one more quadrupole is required. 
For the following a general beam line layout is assumed consisting of four quadrupoles separated by drift lengths:

$$
\text { beam }->q_{4}-1_{4}-q_{3}-1_{3}-q_{2}-1_{2}-q_{1}-1_{1}-\text { point of interest }
$$

The strength of quadrupole 4 is taken as independent parameter and only small changes are considered as described above. The changes of the strengths of the remaining three quadrupoles are then a function of the changes of $q_{4}$ :

$$
\begin{aligned}
& \mathrm{dq}_{3}=\mathrm{f}_{1} \mathrm{dq}_{4} \\
& \mathrm{dq}_{2}=\mathrm{f}_{2} \mathrm{dq}_{4} \\
& \mathrm{dq}_{1}=\mathrm{f}_{1} \mathrm{dq}_{4}
\end{aligned}
$$

For thin lens approximation of the quadrupoles analytic expressions for the coefficients $\mathrm{f}_{1}, \mathrm{f}_{2}$, and $\mathrm{f}_{3}$ can be found :

$$
\begin{aligned}
& f_{1}=-\frac{1_{4}\left(1_{3}+1_{4}+l_{3} 1_{4} q_{3}\right)}{1_{2}\left(1_{2}+1_{3}+1_{2} 1_{3} q_{2}\right)} \\
& f_{2}=\frac{l_{4}\left(l_{2}+l_{3}+1_{4}+l_{2} l_{3} q_{2}+l_{2} l_{4} q_{2}+1_{2} l_{4} q_{3}+l_{3} l_{4} q_{3}+1_{2} l_{3} l_{4} q_{2} q_{3}\right)}{1_{2} l_{3}}
\end{aligned}
$$

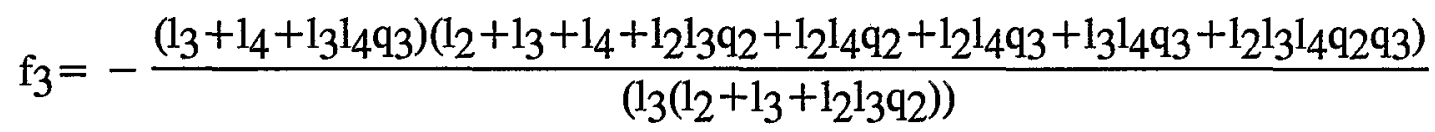

The following table shows the values of these coefficients calculated for section 29 of the HTB line for a nominal beam line setting. The first set of coefficients changes only the horizontal beam parameters whereas the second set changes only the vertical beam parameters.

\begin{tabular}{|l|c|c|c|}
\hline & $\mathrm{f}_{1}$ & $\mathrm{f}_{2}$ & $\mathrm{f}_{3}$ \\
\hline $\begin{array}{l}\text { Change horizontal } \\
\text { beam parameters }\end{array}$ & -1.08 & 0.71 & -0.76 \\
\hline $\begin{array}{l}\text { Change vertical } \\
\text { beam parameters }\end{array}$ & -0.95 & 1.42 & -1.35 \\
\hline
\end{tabular}

Figure 1 shows the calculated beam parameters at the point of interest (injection into Booster) as a function of the percentage change of the strength of quadrupole 4 . The remaining quadrupoles were changed to leave the vertical beam parameters unchanged 
(dashed line) or to leave the horizontal beam parameters unchanged (solid line). The beam transport calculation was done using thick lense matrices for the quadrupoles but using coefficients $f_{1}, f_{2}$, and $f_{3}$ from the table above (thin lens approximation). This explains the small residual slope for those parameters that should remain unchanged.

The method described above is independent of the parameters of the beam. If the initial beam parameters are know then coefficients could be calculated that change $\alpha_{\mathrm{X}}, \beta_{\mathrm{X}}$, $\alpha_{y}$, and $\beta_{y}$ independently using only four quadrupoles. However, this is only useful to the extend that the initial beam parameters remain constant.

\section{References}

1.J.-L. LeMaire, HOPI - On-line Injection Optimization Program, BNL 50741 


\section{alpha-y}

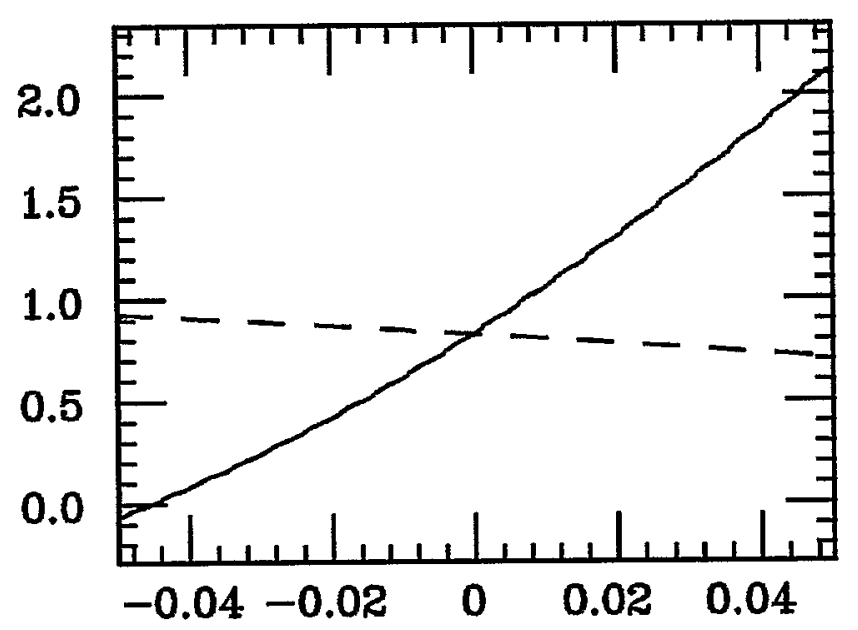

alpha-x

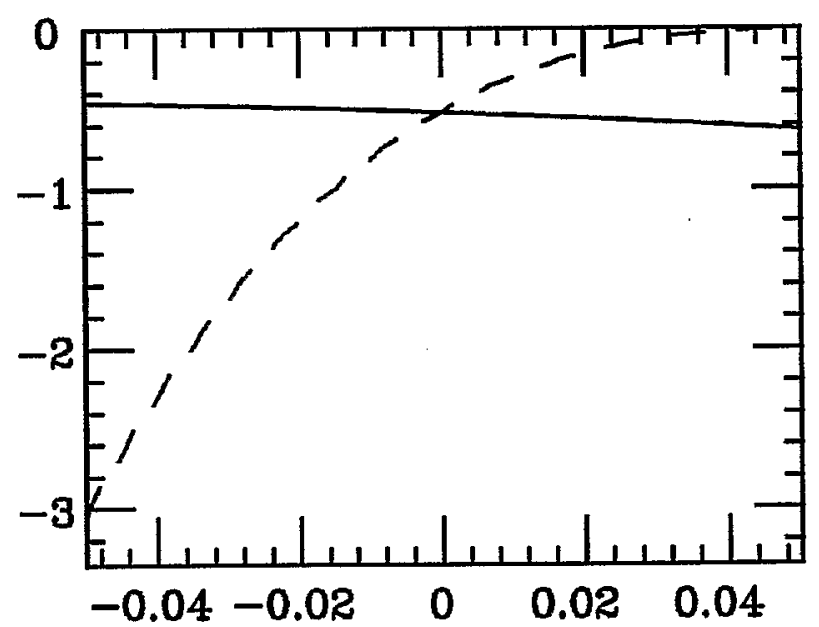

beta-y

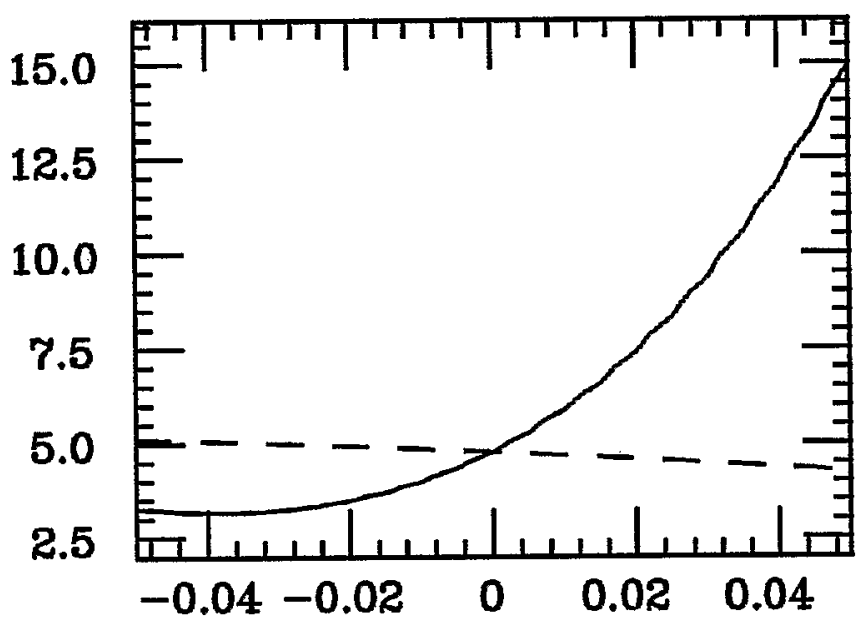

beta $-x$

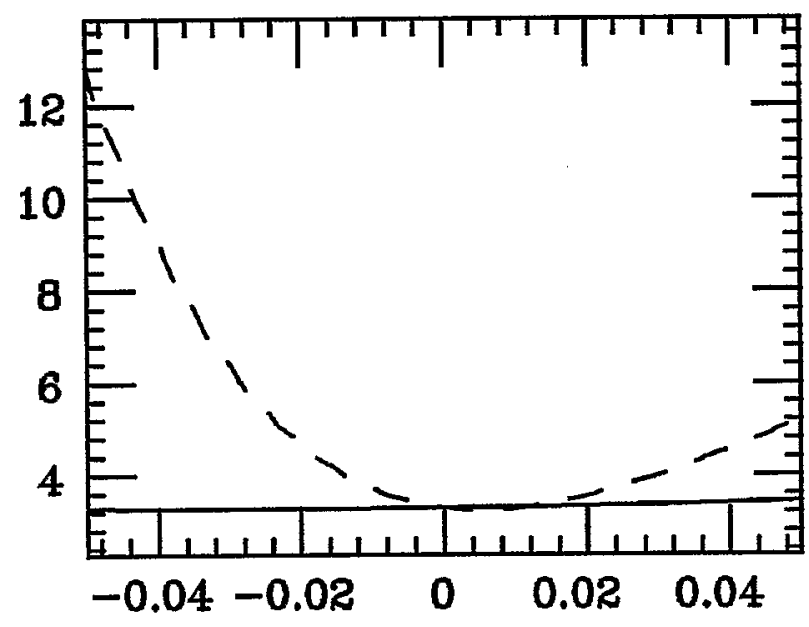

